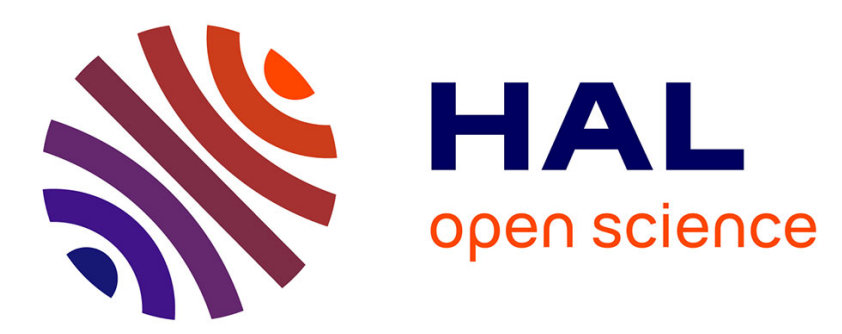

\title{
Constant time-horizon prediction-based stabilization for linear systems with input-dependent input-delay
}

Flore Falcon, Delphine Bresch-Pietri

\section{To cite this version:}

Flore Falcon, Delphine Bresch-Pietri. Constant time-horizon prediction-based stabilization for linear systems with input-dependent input-delay. Conference on Decision and Control, Dec 2021, Austin, TX, United States. hal-03335383

\section{HAL Id: hal-03335383 \\ https://hal.science/hal-03335383}

Submitted on 6 Sep 2021

HAL is a multi-disciplinary open access archive for the deposit and dissemination of scientific research documents, whether they are published or not. The documents may come from teaching and research institutions in France or abroad, or from public or private research centers.
L'archive ouverte pluridisciplinaire HAL, est destinée au dépôt et à la diffusion de documents scientifiques de niveau recherche, publiés ou non, émanant des établissements d'enseignement et de recherche français ou étrangers, des laboratoires publics ou privés. 


\title{
Constant time-horizon prediction-based stabilization for linear systems with input-dependent input-delay
}

\author{
Flore Falcon, Delphine Bresch-Pietri
}

\begin{abstract}
This paper investigates prediction-based stabilization for a class of linear systems subject to input-dependent input delay. The delay under consideration is implicitly defined by an integral relation depending on past values of the input. This situation is frequent in the process industry. We propose here to use a prediction on an horizon equal to the equilibrium value of the delay. By relying on a novel transport Partial Differential Equation (PDE) representation of the delay, encapsulating the input-dependency, we prove local exponential stabilization of the closed-loop system.
\end{abstract}

\section{INTRODUCTION}

Numerous systems involve a time lag resulting from transportation of material. Such a situation is frequent in chemical, process and combustion engineering and occurs, e.g., for airpath regulation in automotive engines [24], [27], control of after-treatment devices in exhaust lines [14], [23], chemical reactors [19], [17] and tubular reactors [35] or, again, flow regulation in mining [37]. Remarkably, in most of these examples, this transport phenomenon depends on the manipulated variable, resulting into an input-dependent delay.

Interestingly enough, this problem is listed as one of the open problems of the field in [32], and has seldom been studied in the literature [16]. Instead, most of the existing approaches ignore this feature and recast the input-dependent delay as a general time-varying delay. The controller design is then performed to cope with a certain level of unstructured delay variations.

In this work, we consider a specific class of structured delays, sometimes called transport delays or hydraulic delays. Such delays are implicitly defined as the solution of an integral relation depending on past values of the input [38]. They are the exact solution of a plug-flow transport equation (see [11], [31]), hence their designation as hydraulic or transport. Their appearance can be traced back to the modeling of combustion instabilities in rocket in [26], [34]. However, up to our knowledge, only a few theoretical studies have investigated control related issues. Notable exceptions are [7] which proposes an heuristic control design in the case of a state delay and [12], [13] which investigate optimal control for a general class of nonlinear systems with both input and state transport delays.

This paper focuses on the design of a prediction-based control law (see [1], [25], [33]) for a linear system subject to such a transport input delay. For a constant delay, predictionbased controllers are now state-of-the-art ([18], [20], [28])

F. Falcon and D. Bresch-Pietri (corresponding author) are with Centre Automatique et Systèmes (CAS), MINES ParisTech, Université PSL, 75006 Paris, France Email: delphine.bresch-pietri@mines-paristech.fr and have been lately extended to time-varying ones (see [30], [5]) in a large variety of contexts ([36], [2], [3], [4], [22]). In such cases, to compensate for the varying delay, the prediction horizon should match the future delay value and has to be obtained by a careful design. However, when the delay depends on the input, things are getting intricate. Indeed, determining such a prediction horizon becomes an implicit question, due to the mutual dependency between the delay and the (current and past) input values. While exact delay compensation can be achieved when the delay depends only on the current or one delayed value of the input ([15], [6]), for a transport delay, determining a suitable horizon prediction may not be feasible.

For this reason, in lieu of seeking exact delay compensation, we propose to follow a robust compensation approach. Our control design relies on a state prediction over a constant horizon, chosen as the asymptotic delay value. Note that this horizon differs from the one chosen in our previous works on the topic [8], [9], [10]. Regarding stability analysis, the main novelty of this work consists in the introduction of a Partial Differential Equation (PDE) representation of the transport delay in which the propagation speed is the input itself. This enables us to significantly simplify the stability analysis, compared to the two-step procedures which was previously proposed in [8]. Applying then a standard backstepping transformation, we prove local stabilization towards a given equilibrium. This is the main contribution of this work.

This paper is organized as follows. In Section II, we present the problem under consideration along with the proposed control design and state the main result of the paper. Then, in Section III, we reformulate the transport delay as a transport hyperbolic PDE the speed of which is the input. Section IV details the stability analysis. Finally, Section V illustrates the control design of the paper with numerical simulations.

Notations and definitions In the following, $\|\cdot\|$ stands for the Euclidean norm on $\mathbb{R}^{n}$. $\|f(t)\|_{2}$ represents the $L_{2}$-norm of a spatially distributed variable $f:(x, t) \in[0 ; 1] \times \mathbb{R}^{m} \rightarrow \mathbb{R}$ and is defined as

$$
\|f(t)\|_{2}=\sqrt{\int_{0}^{1}\|f(x, t)\|^{2} d x}
$$

We denote $f_{x}$ the partial derivative of the function $f$ with respect to the variable $x$, and $f_{t}$ the partial derivative of $f$ with respect to the variable $t$. The operator $\mathrm{Sat}_{I}$ of a scalar variable over an interval $I$ stands for the saturation operator onto the interval I. 


\section{PRoBlem STATEMENT AND CONTROL DESIGN}

Consider the following (potentially unstable) linear inputdelay system

$$
\dot{X}(t)=A X(t)+B \varphi(t-D(t))
$$

where $X \in \mathbb{R}^{n}$ and $\varphi \in \mathbb{R}$ are the state and the input of the system, respectively, $A \in \mathscr{M}_{n}(\mathbb{R})$ and $B \in \mathbb{R}^{n}$ are the dynamic matrices and the time-varying input delay $D(t)$ is defined implicitly in terms of past values of the input according to the following implicit integral relation

$$
\int_{t-D(t)}^{t} \varphi(s) d s=1
$$

with

$$
\varphi(t)=\operatorname{Sat}_{[\underline{U}, \bar{U}]}(U(t))
$$

in which $0<U<\bar{U}$. We refer to this class of delay as transport delay, as the exact solution of a plug-flow transport equation [31] where the propagation speed is the input $\varphi$ (see [11] for a detailed overview of the properties of this class of delays). Note that the lower saturation in (3) aims at guaranteeing that the delay is upper-bounded and does not tend to infinity.

The control objective it to stabilize the system towards an equilibrium value $X^{r}$ corresponding to a control reference $U^{r} \in(\underline{U}, \bar{U})$. With this aim in view, we consider the prediction-based control law

$$
U(t)=U_{r}-K X^{r}+K e^{D^{r} A} X(t)+K \int_{t-D^{r}}^{t} e^{A(t-s)} B U(s) d s
$$

where $D^{r}=1 / U^{r}$ corresponds to the nominal equilibrium delay value and $K \in \mathscr{M}_{1 \times n}(\mathbb{R})$ is such that $A+B K$ is Hurwitz.

Notice that this choice is directly inspired by the constant delay case. Indeed, if the delay were constant and equal to $D^{r}$, equation (4) would then correspond to the value $U^{r}+K\left(X\left(t+D^{r}\right)-X^{r}\right)$, that is, to an exact prediction of the system state $D^{r}$ units of time ahead.

Theorem 1: Consider the closed-loop system consisting of the system (1)-(3) and the control law (4). Define the functional

$$
\begin{aligned}
\Upsilon(t) & =\left\|X(t)-X_{r}\right\|^{2}+\int_{t-\max \left\{D(t), D^{r}\right\}}^{t}\left(U(s)-U_{r}\right)^{2} d s \\
& +\left(D^{r}\right)^{2} \int_{t-\bar{D}}^{t} \dot{U}(s)^{2} d s
\end{aligned}
$$

with $\bar{D}=1 / \underline{U}$ the maximum delay value. There exists $r^{*}>0$ such that, if $\Upsilon(0) \leq r^{*}$, then $\varphi(t)=U(t) \in(\underline{U}, \bar{U})$ for all $t \geq 0$ and the closed-loop system exponentially converges to $\left(X^{r}, U^{r}\right)$ in the sense of the norm $\Upsilon$, that is, there exist $R>0$ and $\theta>0$ such that

$$
\Upsilon(t) \leq R \Upsilon(0) e^{-\theta t}, \quad t \geq 0
$$

This result states that, if the initial conditions are sufficiently close to the desired equilibrium, exponential stabilization at this equilibrium is achieved. This is consistent with the choice of the control law (4). Indeed, as the prediction horizon corresponds to the asymptotic delay value, this prediction is likely to be sufficiently accurate only close to the equilibrium. Note that this local condition is also required in [8], [10] and [6]. Compared, e.g., to [8] which uses the current delay value as a prediction horizon, the proposed design is likely to yield somehow relatively poorer transient performances. However, it presents the numerical advantages of not requiring to compute the current delay value and of involving an integral over a constant fixed horizon.

III. TRANSPORT PDE REPRESENTATION OF THE INTEGRAL RELATION (3) AND EXTENDED ERROR SYSTEM

In view of stability analysis, let us introduce a new representation of the delay phenomenon by defining the distributed variable

$$
u(x, t)=\varphi(t-\delta(x, t))
$$

for $x \in[0,1]$, with $\delta(x, t)$ defined as the unique solution (see [11] for details) to

$$
\int_{t-\delta(x, t)}^{t} \varphi(s) d s=1-x
$$

Taking time- and space-derivatives of (6) and (7), one easily obtains that system (1)-(3) can be equivalently represented by the following ODE-PDE system

$$
\begin{aligned}
\dot{X}(t) & =A X(t)+B u(0, t) \\
u_{t}(x, t) & =u(1, t) u_{x}(x, t) \\
u(1, t) & =\varphi(t)
\end{aligned}
$$

Notice that this representation simply corresponds to the plug-flow equation [31] mentioned above. Contrary to [15] which involved the value at the unactuated boundary, here, the transport speed is the current input. Also, it is worth underlying that the transport speed of this equation is strictly positive as well as uniformly bounded from above and below, due to the saturation (3).

The following lemma formally expresses this equivalence.

Lemma 1: Consider the delay $D$ defined in (2). Then,

$$
\frac{1}{\bar{U}}\|u(t)\|_{2}^{2} \leq \int_{t-D(t)}^{t} \varphi(s)^{2} d s \leq \frac{1}{\underline{U}}\|u(t)\|_{2}^{2}
$$

Proof: From (6), with a change of variable and noticing that $\delta(0, t)=D(t)$ and $\delta(1, t)=0$, one obtains that

$$
\|u(t)\|_{2}^{2}=\int_{t-D(t)}^{t}-\frac{1}{\delta_{x}\left(\sigma^{-1}(s), t\right)} \varphi(s)^{2} d s
$$

in which, for a fixed $t \in \mathbb{R}, \sigma: x \in[0,1] \mapsto t-\delta(x, t)$ is a continuous, decreasing and, therefore, invertible function. Furthermore, taking a space-derivative of (7), $-\frac{1}{\delta_{x}(x, t)}=$ $\varphi(t-\delta(x, t)) \in[\underline{U} ; \bar{U}]$. The result directly follows.

Besides, we also introduce the stabilization error variables $\tilde{X}(t)=X(t)-X_{r}, \tilde{\varphi}(t)=\varphi(t)-U_{r}, \tilde{U}(t)=U(t)-U_{r}$ and $\tilde{u}(x, t)=u(x, t)-U_{r}$. Finally, we define the distributed variables

$$
\left\{\begin{array}{l}
\hat{u}(x, t)=\tilde{U}\left(t+D^{r}(x-1)\right) \\
e(x, t)=\tilde{u}(x, t)-\hat{u}(x, t)
\end{array}\right.
$$


The corresponding extended system writes

$$
\left\{\begin{aligned}
\dot{\tilde{X}}(t) & =A \tilde{X}(t)+B(e(0, t)+\hat{u}(0, t)) \\
D^{r} \hat{u}_{t}(x, t) & =\hat{u}_{x}(x, t) \\
\hat{u}(1, t) & =\tilde{\varphi}(t) \\
D^{r} e_{t}(x, t) & =e_{x}(x, t)+D^{r} \hat{u}(1, t)\left(e_{x}(x, t)+\hat{u}_{x}(x, t)\right) \\
e(1, t) & =0
\end{aligned}\right.
$$

We are now ready to carry out the stability analysis.

\section{STABILITY ANALYSis}

As a first step in our analysis, we assume that the control input is not saturated (Subsections IV-A and IV-B). The effect of this saturation is later analyzed in Subsection IV-C which contains the proof of Theorem 1.

\section{A. Backstepping transformation}

In view of Lyapunov analysis, define the backstepping transformation

$$
w(x, t)=\hat{u}(x, t)-K e^{D^{r} A x} \tilde{X}(t)-K D^{r} \int_{0}^{x} e^{A D^{r}(x-y)} B \hat{u}(y, t) d y
$$

Lemma 2: Assume that the closed-loop system consisting of the system (1)-(3) and the control law (4) results into an unsaturated control law, that is, $U(t) \in(\underline{U}, \bar{U})$ for all $t \geq 0$. Then, the backstepping transformation (14) along with the control law (4) transform system (13) into the target system

$$
\begin{aligned}
\dot{\tilde{X}}(t) & =(A+B K) \tilde{X}(t)+B(e(0, t)+w(0, t)) \\
D^{r} w_{t} & =w_{x}-D^{r} K e^{D^{r} A x} B e(0, t) \\
w(1, t) & =0 \\
D^{r} e_{t}(x, t) & =e_{x}(x, t) \\
+ & D^{r} h(\tilde{X}(t), w(\cdot, t))\left(e_{x}(x, t)+g\left(\tilde{X}(t), w(\cdot, t), w_{x}(\cdot, t), x\right)\right. \\
e(1, t) & =0
\end{aligned}
$$

in which

$$
\begin{gathered}
h(\tilde{X}(t), w(\cdot, t))=K\left[e^{D^{r}(A+B K)} \tilde{X}(t)\right. \\
\left.+\int_{0}^{1} e^{D^{r}(A+B K)(1-y)} B w(y, t) D^{r} d y\right] \\
g\left(\tilde{X}(t), w(\cdot, t), w_{x}(\cdot, t), x\right)=w_{x}(x, t)+K D^{r}(B w(x, t) \\
\left.+(A+B K)\left[e^{D^{r}(A+B K) x} \tilde{X}(t)+D^{r} \int_{0}^{x} e^{D^{r}(A+B K)(x-y)} B w(y, t) d y\right]\right)
\end{gathered}
$$

In addition, the space-derivative of the backstepping variable satisfies

$$
\begin{aligned}
D^{r} w_{x t}(x, t) & =w_{x x}-\left(D^{r}\right)^{2} K A e^{D^{r} A x} B e(0, t) \\
w_{x}(1, t) & =D^{r} K^{D^{r} A} B e(0, t)
\end{aligned}
$$

Proof: Assuming that, for $t \geq 0, U(t) \in(\underline{U}, \bar{U})$ implies that $\varphi(t)=U(t)$ and, thus, the boundary condition (17) follows. Besides, one concludes that $\tilde{\varphi}(t-D(t))=\hat{u}(0, t)=$ $w(0, t)+K \tilde{X}(t)$ which gives (15). The dynamics of the bacstepping transformation can be straightforwardly obtained taking time- and space-derivatives of (14). Furthermore, the expressions (20)-(21) of the functions $h$ and $g$ involved in (18) are obtained with the inverse of the backstepping transformation (14) which is

$$
\begin{aligned}
\hat{u}(x, t) & =w(x, t)+K e^{D^{r}(A+B K) x} \tilde{X}(t) \\
& +K \int_{0}^{x} e^{D^{r}(A+B K)(x-y)} B w(y, t) D^{r} d y
\end{aligned}
$$

Finally, taking a space-derivative of (16), the dynamics (22)(23) follows.

\section{B. Lyapunov analysis}

Consider the Lyapunov functional candidate

$$
\begin{aligned}
V(t) & =\tilde{X}^{T} P \tilde{X}+b_{w} D^{r} \int_{0}^{1}(1+x) w(x, t)^{2} d x \\
& +b_{e} D^{r} \int_{0}^{1}(1+x) e(x, t)^{2} d x+b_{w_{x}} D^{r} \int_{0}^{1}(1+x) w_{x}(x, t)^{2} d x
\end{aligned}
$$

with $b_{w}>0, b_{e}>0, b_{w_{x}}>0$ positive constants to be chosen later and $P$ the symmetric definite positive solution to the Lyapunov equation $(A+B K)^{T} P+P(A+B K)=-Q$ for a given symmetric definite positive matrix $Q$.

Lemma 3: Assume that the closed-loop system consisting of the system (1)-(3) and the control law (4) results into an unsaturated control law, that is, $U(t) \in(\underline{U}, \bar{U})$ for all $t \geq 0$. There exist positive constants $b_{e}, b_{w}$ and $b_{w_{x}}$ such that

$$
\dot{V}(t) \leq-\left(\eta_{1}-\eta_{2} \sqrt{V(t)}\right) e(0, t)^{2}-\left(\eta_{3}-\eta_{4} \sqrt{V(t)}\right) V(t)
$$

with $\eta_{1}, \eta_{2}, \eta_{3}, \eta_{4}$ positive constants.

Proof: As Lemma 2 holds, taking a time-derivative of (25), using the dynamics (15)-(23) and performing integrations by parts, one obtains

$$
\begin{aligned}
\dot{V}(t)= & -\tilde{X}(t)^{T} Q \tilde{X}(t)+2 \tilde{X}(t)^{T} P B(w(0, t)+e(0, t)) \\
- & b_{w} w(0, t)^{2}-b_{w}\|w(t)\|_{2}^{2} \\
- & 2 K b_{w} D^{r} \int_{0}^{1}(1+x) w(x, t) e^{D^{r} A x} B e(0, t) d x \\
- & b_{e}\left[1+D^{r} h(\tilde{X}(t), w(\cdot, t))\right]\left(e(0, t)^{2}+\|e(t)\|_{2}^{2}\right) \\
+ & 2 b_{e} D^{r} h(\tilde{X}(t), w(\cdot, t)) \\
& \times \int_{0}^{1}(1+x) e(x, t) g\left(\tilde{X}(t), w(\cdot, t), w_{x}(\cdot, t), x\right) d x \\
+ & 2 b_{w_{x}} w_{x}(1, t)^{2}-b_{w_{x}} w_{x}(0, t)^{2}-b_{w_{x}}\left\|w_{x}(t)\right\|_{2}^{2} \\
- & 2 K b_{w_{x}} D^{r} \int_{0}^{1}(1+x) w_{x}(x, t) D^{r} A e^{D^{r} A x} B e(0, t) d x
\end{aligned}
$$

Using the expressions of $h$ and $g$ in (20)-(21), the boundary condition (23) and Young's inequality, there exist $\gamma_{1}>0$ and 
$\gamma_{2}>0$ such that

$$
\begin{aligned}
& \dot{V}(t) \leq-\frac{\lambda_{\min }(Q)}{2}\|\tilde{X}(t)\|^{2}-b_{w}\left(1-M_{w} \gamma_{1}\right)\|w(t)\|_{2}^{2} \\
& \quad-b_{e}\|e(t)\|_{2}^{2}-b_{w_{x}}\left(1-M_{w_{x}} \gamma_{2}\right)\left\|w_{x}(t)\right\|_{2}^{2} \\
& \quad+b_{e} D^{r}\left(M_{0}\|\tilde{X}(t)\|+M_{1}\|w(t)\|_{2}\right)\|e(t)\|_{2}^{2} \\
& \quad-\left(b_{e}-\frac{2}{\lambda_{\min }(Q)}\|P B\|^{2}-2 b_{w_{x}}\left(D^{r}\right)^{2}\left(K e^{D^{r} A} B\right)^{2}-\frac{b_{w} M_{w}}{\gamma_{1}}\right. \\
& \left.\quad-\frac{b_{w_{x}} M_{w_{x}}}{\gamma_{2}}-b_{e} D^{r}\left(M_{0}\|\tilde{X}(t)\|+M_{1}\|w(t)\|_{2}\right)\right) e(0, t)^{2} \\
& \quad-\left(b_{w}-\frac{2}{\lambda_{\min }(Q)}\|P B\|^{2}\right) w(0, t)^{2}-b_{w_{x}} w_{x}(0, t)^{2} \\
& \quad+2 b_{e} D^{r}\left(M_{0}\|\tilde{X}(t)\|+M_{1}\|w(t)\|_{2}\right)\left(4\left\|w_{x}(t)\right\|_{2}\|e(t)\|_{2}\right. \\
& \left.\quad+M_{2}\|\tilde{X}(t)\|_{2}\|e(t)\|_{2}+M_{4}\|w(t)\|_{2}\|e(t)\|_{2}\right)
\end{aligned}
$$

in which

$$
\begin{aligned}
M_{w}= & \left(D^{r}\right)^{2}\left\|x \mapsto(1+x) K e^{D^{r} A x}\right\|_{2} \\
M_{w_{x}}= & \left(D^{r}\right)^{3}\left\|x \mapsto(1+x) K A e^{D^{r} A x}\right\|_{2} \\
M_{0}= & n \max _{j}\left(\sum_{i=1}^{n}\left|K_{i}\right|\left|E_{i j}\right| \text { with } E=e^{D^{r}(A+B K)}\right. \\
M_{1}= & D^{r}\left\|y \mapsto K e^{D^{r}(A+B K)(1-y)} B\right\|_{2} \\
M_{2}= & 4 D^{r} n\left\|x \mapsto \max _{j}\left(\sum_{i=1}^{n}\left|K_{i} \| F_{i j}(x)\right|\right)\right\| \\
& \text { with } F=(A+B K) e^{D^{r}(A+B K) x} \\
M_{3}= & \left\|x \mapsto(1+x)\left(D^{r}\right)^{2} f(x)\right\|_{2} \\
& \text { with } f(x)=\left\|y \mapsto K(A+B K) e^{D^{r}(A+B K)(x-y)} B\right\|_{2} \\
M_{4}= & M_{3}+4 D^{r}|K B|
\end{aligned}
$$

Using again Young's inequality and rearranging terms, it follows that

$$
\begin{aligned}
& \dot{V}(t) \leq-\frac{\lambda_{\min }(Q)}{2}\|\tilde{X}(t)\|^{2}-b_{w}\left(1-M_{w} \gamma_{1}\right)\|w(t)\|_{2}^{2} \\
& \quad-b_{e}\|e(t)\|_{2}^{2}-b_{w_{x}}\left(1-M_{w_{x}} \gamma_{2}\right)\left\|w_{x}(t)\right\|_{2}^{2} \\
& \quad-\left(b_{e}-\frac{2}{\lambda_{\min }(Q)}\|P B\|^{2}-\frac{b_{w} M_{w}}{\gamma_{1}}-2 b_{w_{x}}\left(D^{r}\right)^{2}\left(K e^{D^{r} A} B\right)^{2}\right. \\
& \left.\quad-\frac{b_{w_{x}} M_{w_{x}}}{\gamma_{2}}-b_{e} D^{r}\left(M_{0}\|\tilde{X}(t)\|+M_{1}\|w(t)\|_{2}\right)\right) e(0, t)^{2} \\
& \quad-\left(b_{w}-\frac{2}{\lambda_{\min }(Q)}\|P B\|^{2}\right) w(0, t)^{2}-b_{w_{x}} w_{x}(0, t)^{2} \\
& \quad+b_{e} D^{r}\left(\left(M_{0}+M_{1} M_{2}\right)\|\tilde{X}(t)\|+\left(M_{1}+M_{0} M_{4}\right)\|w(t)\|_{2}\right. \\
& \left.\quad+4\left(M_{0}+M_{1}\right)\left\|w_{x}(t)\right\|_{2}\right)\|e(t)\|_{2}^{2}+b_{e} D^{r}\left(4 M_{0}\left\|w_{x}(t)\right\|_{2}\right. \\
& \left.\quad+2 M_{0} M_{2}\|e(t)\|_{2}+M_{0} M_{4}\|w(t)\|_{2}\right)\|\tilde{X}(t)\|^{2}+b_{e} D^{r} \\
& \quad \times\left(4 M_{1}\left\|w_{x}(t)\right\|_{2}+M_{2} M_{1}\|\tilde{X}(t)\|+M_{4} M_{1}\|e(t)\|_{2}\right)\|w(t)\|_{2}^{2}
\end{aligned}
$$

We choose $\gamma_{1}, \gamma_{2}, b_{w}$ and finally $b_{e}$ and $b_{w}$ such that

$$
\begin{aligned}
1-M_{w} \gamma_{1} & >0 \\
1-M_{w_{x}} \gamma_{2} & >0 \\
b_{w} & >\frac{2}{\lambda_{\min }(Q)}\|P B\|_{2}^{2} \\
b_{e}+\frac{b_{w_{x}} M_{w_{x}}}{\gamma_{2}} & >\eta_{1} \\
\triangleq & \frac{2}{\lambda_{\min }(Q)}\|P B\|_{2}^{2}-\frac{b_{w} M_{w}}{\gamma_{1}}-2 b_{w_{x}}\left(D^{r}\right)^{2}\left(K e^{D^{r} A} B\right)^{2}
\end{aligned}
$$

Finally, noticing that

$$
\mu_{m} V_{0}(t) \leq V(t) \leq \mu_{M} V_{0}(t)
$$

with $V_{0}(t)=\left(\|\tilde{X}(t)\|^{2}+\|w(t)\|_{2}^{2}+\|e(t)\|_{2}^{2}+\left\|w_{x}(t)\right\|_{2}^{2}\right)$, $\mu_{m}=\min \left\{\lambda_{\min }(P) / 2, D^{r} b_{w}, D^{r} b_{e}, D^{r} b_{w_{x}}\right\}$ and $\mu_{M}=$ $\max \left\{\lambda_{\max }(P) / 2,2 D^{r} b_{w}, 2 D^{r} b_{e}, 2 D^{r} b_{w_{x}}\right\}$, the expected result follows defining

$$
\begin{aligned}
& \eta_{2}=4 \frac{b_{e} D^{r} \max \left(M_{0}, M_{1}\right)}{\sqrt{\mu_{m}}} \\
& \eta_{3}=\frac{1}{\mu_{M}} \min \left\{\frac{\lambda_{\min }(Q)}{2}, b_{w}\left(1-M_{w} \gamma_{1}\right), b_{e}, b_{w_{x}}\left(1-M_{w_{x}} \gamma_{2}\right)\right\} \\
& \eta_{4}=4 \frac{M}{\mu_{m} \sqrt{\mu_{m}}}
\end{aligned}
$$

with $M=b_{e} D^{r} \max \left(\left\{M_{0}+M_{1} M_{2} ; M_{1}+M_{0} M_{4} ; 4\left(M_{0}+\right.\right.\right.$ $\left.\left.\left.M_{1}\right) ; 4 M_{0} ; 2 M_{0} M_{2} ; M_{0} M_{4} ; 4 M_{1} ; M_{2} M_{1} ; M_{4} M_{1}\right\}\right)$.

\section{Proof of Theorem 1}

We start this proof by noticing that, if $U(t) \in(\underline{U}, \bar{U})$ for all $t \in \mathbb{R}$, that is, the control law is not saturated, there exist $a, b>0$ such that

$$
a V(t) \leq \Upsilon(t) \leq b V(t), \quad t \geq 0
$$

in which $\Upsilon$ defined in (5) can be straightforwardly written in terms of $\tilde{X}$ and $\tilde{U}$. Equation (43) follows from Lemma 1, the backstepping transformation (14), its inverse (24), and the fact that, using a change of variable,

$$
D^{r} \int_{t-D^{r}}^{t} \dot{U}(s)^{2} d s=\left\|\hat{u}_{x}(t)\right\|^{2}
$$

Second, we prove that there exists $c>0$ such that $|\tilde{U}(t+s)| \leq$ $c \sqrt{V(t)}$, for all $t \geq 0$ and $s \in[-\bar{D}, 0]$. Indeed, using (4), one gets

$$
\begin{aligned}
\tilde{U}(t+s)=\tilde{U}(t)+\int_{t}^{t+s} \dot{\tilde{U}}(\xi) d \xi \\
=K e^{D^{r} A} \tilde{X}(t)+K \int_{t-D^{r}}^{t} e^{A(t-\xi)} B \tilde{U}(\xi) d \xi \\
\quad-\int_{t+s}^{t} \dot{\tilde{U}}(\xi) d \xi, \quad t \geq 0, \quad s \in[-\bar{D}, 0]
\end{aligned}
$$

Applying Cauchy-Schwarz's inequality and using (43), the result then follows for

$$
c=3 \sqrt{b \max \left\{N_{1}^{2}, \frac{N_{2}^{2}}{D^{r}}, \frac{\bar{D}}{\left(D^{r}\right)^{2}}\right\}}
$$


in which

$$
\begin{aligned}
& N_{1}=n \max _{j}\left(\sum_{i=1}^{n}\left|K_{i}\right|\left|H_{i j}\right|\right) \text { where } H=e^{D^{r} A} \\
& N_{2}=D^{r}\left\|y \longrightarrow K e^{A D^{r}(1-y)} B\right\|_{2}
\end{aligned}
$$

We are now ready to conclude the proof of Theorem 1 .

Define $R_{0}=\min \left\{U^{r}-\underline{U}, \bar{U}-U^{r}\right\}$ and

$$
r^{\star}=a \min \left\{\min \left\{\frac{\eta_{1}}{\eta_{2}}, \frac{\eta_{3}}{\eta_{4}}\right\}^{2}, \frac{R_{0}^{2}}{c^{2}}\right\}-\varepsilon
$$

in which $\eta_{1}, \eta_{2}, \eta_{3}$ and $\eta_{4}$ are defined in Lemma 3 and $\varepsilon$ is a positive constant such that $r^{*}>0$. Assume that $\Upsilon(0) \leq$ $r^{*}$. Then, from (43), $V(0)<R_{0}^{2} / c^{2}$, which implies from the definition of $c$ that, for $s \in[-\bar{D}, 0],|\tilde{U}(s)|<R_{0}$ and, thus, that $U(s) \in(\underline{U}, \bar{U})$. Consequently, Lemma 3 holds and, as $V(0)<\min \left\{\frac{\eta_{1}}{\eta_{2}}, \frac{\eta_{3}}{\eta_{4}}\right\}^{2}$, there exist $\theta>0$ such that

$$
V(t) \leq V(0) e^{-\theta t}, \quad t \geq 0
$$

Consequently, $|\tilde{U}(t)| \leq c \sqrt{V(t)} \leq c \sqrt{V(0)}<R_{0}$ which implies that $U(t) \in(\underline{U}, \bar{U})$ for all $t \geq 0$ and the result follows with $R=b / a$, using (43).

\section{Simulation RESUlTS}

To illustrate the merits of our control design and to ease the understanding of the local stabilization result stated in Theorem 1, we consider in this section a simple unstable scalar plant $(n=1)$, with $A=B=1$ and $\underline{U}=0.1$. As the upper value $\bar{U}$ of the saturation only plays a technical role in the previous proof, it is thus chosen arbitrarily large in simulations. We pick $X^{r}=-1$. The corresponding equilibrium values for the input and the delay are then $U^{r}=1$ and $D^{r}=1$. The initial conditions are selected as $X(0)=-1.2$ and $U(s)=1$ for $s \in[-\bar{D}, 0)$. Finally, we select the $K$ such that $A+B K=1+K$ is Hurwitz, that is, $K<-1$. Discretization of the integral involved in the prediction (4) is performed with a trapezoidal approximation (see [21], [29] for details on the numerical challenges associated with this discretization).

Fig. 1 depicts the time-evolution of the closed-loop system, both for the proposed prediction-based controller and for a proportional one using the same feedback gain $K=-1.1$, for the sake of comparison. One can observe that the proportional controller fails to stabilize the system, due to the lower saturation which limits its stabilization capabilities. On the other hand, stabilization towards the desired equilibrium is achieved with the proposed prediction-based controller. Although the input-delay is only robustly compensated, as can be observed by a few number of oscillations visible in the control signal, the anticipation feature of the prediction enables convergence to the reference.

Theorem 1 states a local result, by requiring the initial condition to be close enough to the targeted equilibrium. Indeed, simulations performed for initial conditions much further away from the reference resulted into unstable trajectories. According to the proof provided in the previous
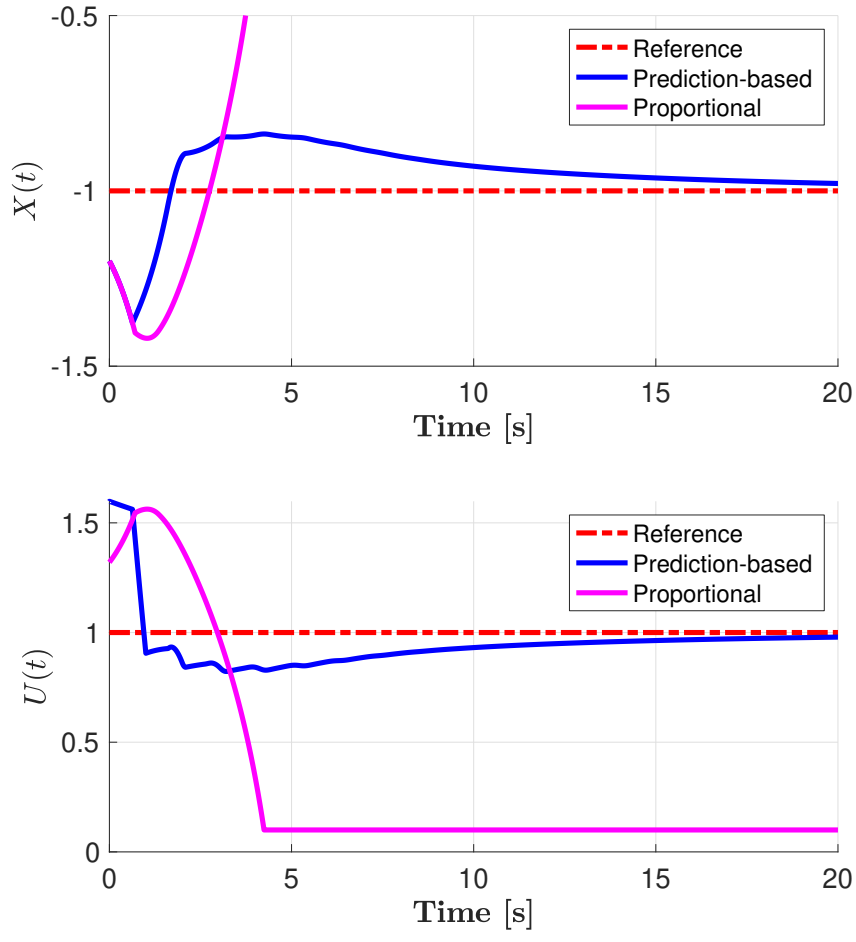

Fig. 1. Time-evolution of the closed-loop system (1)-(4) with $A=B=1$, $\underline{U}=0.1$ and $X^{r}=-1$. Initial conditions are $X(0)=-1.2$ and $U(s)=1$ for $s \in[-\bar{D}, 0)$. The feedback gain is picked as $K=-1.1$. Closed-loop results with a proportional controller using the same gain are also provided.

section, the radius of this basin of attraction depends on the magnitude of the feedback gain. Indeed, intuitively, by increasing the feedback gain, the transient values of the closed-loop system are more likely to steer away from the equilibrium, limiting thus the validity of the prediction but also increasing the risk of saturating the input. Hence, for a given initial condition, this condition is likely to limit the magnitude of the gain to be chosen and could therefore be interpreted as a small-gain condition. Fig. 2 illustrates this limit. Indeed, one can observe that picking $K \leq-1.13$ results into a diverging closed-loop behaviour. Therefore, a tradeoff between transient performances and stability has to be reached.

\section{CONCLUSION}

In this paper, we proposed a prediction-based control design for linear systems subject to a transport input delay. The prediction horizon was selected as the nominal delay value corresponding to the targeted reference. Using a novel transport PDE and backstepping, we proved local exponential stabilization towards the equilibrium. Numerical simulations performed on a scalar example stressed out the crucial rule played by the feedback gain in the size of the basin of attraction.

This point is likely to be improved using the current value of the delay as prediction horizon, as performed in our previous works. Indeed, one could reasonably expect that, by using the current delay value instead of its asymptotic 

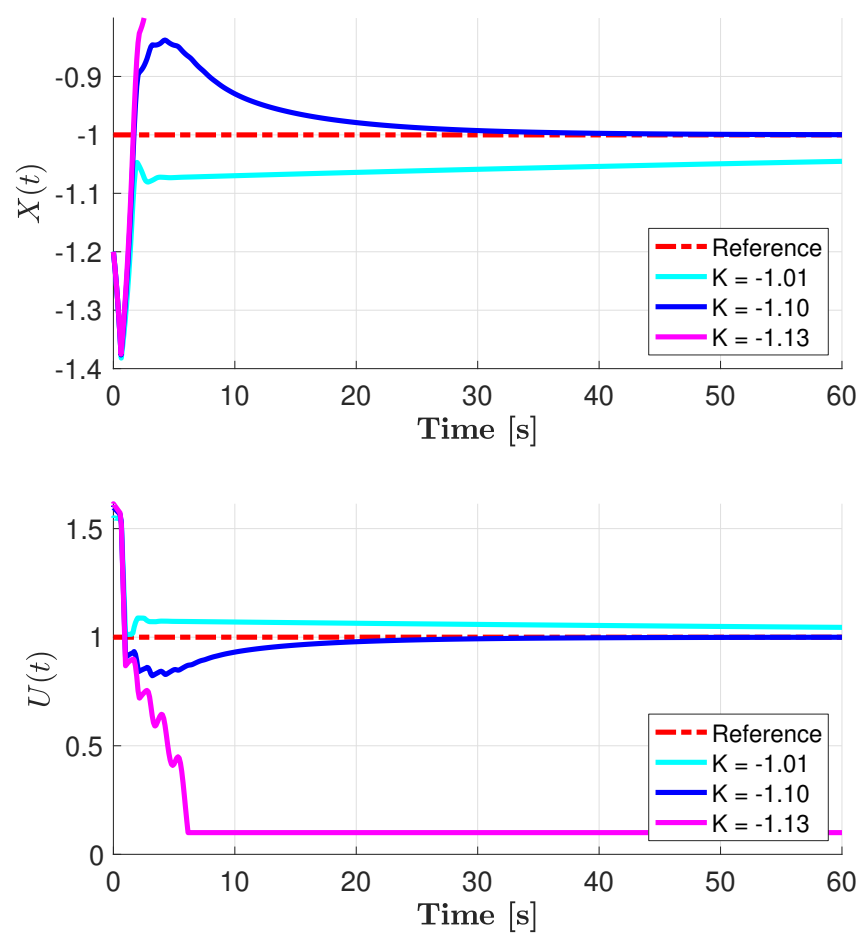

Fig. 2. Time-evolution of the closed-loop system (1)-(4) with $A=B=1$, $\underline{U}=0.1$ and $X^{r}=-1$ and for different values of the feedback gain $K$. Initial conditions are $X(0)=-1.2$ and $U(s)=1$ for $s \in[-\bar{D}, 0)$.

one, such a prediction would be more accurate and yield better closed-loop performances. This intuition seems to be confirmed by the simulations performed in [8]. However, the bakstepping transformation corresponding to such a control law and the transport PDE representation introduced in the present paper is not a Volterra integral equation, but rather a Fredolhm one, for which an inverse transformation may not exist. This and extension of the design to nonlinear systems are directions of future works.

\section{REFERENCES}

[1] Z. Artstein. Linear systems with delayed controls: a reduction. IEEE Transactions on Automatic Control, 27(4):869-879, 1982.

[2] N. Bekiaris-Liberis, M. Jankovic, and M. Krstic. Compensation of state-dependent state delay for nonlinear systems. Systems \& Control Letters, 61(8):849-856, 2012.

[3] N. Bekiaris-Liberis and M. Krstic. Compensation of state-dependent input delay for nonlinear systems. In Conference on Decision and Control, 2011

[4] N. Bekiaris-Liberis and M. Krstic. Nonlinear control under delays that depend on delayed states. European Journal on Control, Special Issue for the ECC13, 19:389-398, 2013.

[5] N. Bekiaris-Liberis and M. Krstic. Nonlinear Control Under Nonconstant Delays, volume 25. Society for Industrial and Applied Mathematics, 2013.

[6] N. Bekiaris-Liberis and M. Krstic. Compensation of transport actuator dynamics with input-dependent moving controlled boundary. IEEE Transactions on Automatic Control, 63(11):3889-3896, 2018.

[7] J. Bendtsen and M. Krstic. Control of thermodynamical system with input-dependent state delays. In 52nd IEEE Conference on Decision and Control, pages 300-305. IEEE, 2013.

[8] D. Bresch-Pietri, J. Chauvin, and N. Petit. Prediction-based stabilization of linear systems subject to input-dependent input delay of integral-type. IEEE Transactions on Automatic Control, 59:23852399, 2014.
[9] D. Bresch-Pietri and K. Coulon. Prediction-based control of moisture in a convective flow. In Proc. of the European Control Conference, pages $43-48,2015$.

[10] D. Bresch-Pietri and F. Di Meglio. Prediction-based control of linear input-delay system subject to state-dependent state delay-application to suppression of mechanical vibrations in drilling. Proc. of the 2 nd IFAC Workshop on Control of Systems Governed by Partial Differential Equations, 49(8):111-117, 2016.

[11] D. Bresch-Pietri and N Petit. Implicit integral equations for modeling systems with a transport delay, chapter in Recent results on time-delay systems: analysis and control. E. Witrant., O. Sename, L. Dugard and E. Fridman (eds), Series Advances in Delay and Dynamics, Springer, 2016.

[12] C.-H. Clerget and N. Petit. Dynamic optimization of processes with time varying hydraulic delays. Journal of Process Control, 83:20-29, 2019.

[13] C.-H. Clerget and N. Petit. Optimal control of systems subject to input-dependent hydraulic delays. IEEE Transactions on Automatic Control, 66(1):245-260, 2020.

[14] C. Depcik and D. Assanis. One-dimensional automotive catalyst modeling. Progress in energy and combustion science, 31(4):308369, 2005.

[15] M. Diagne, N. Bekiaris-Liberis, and M. Krstic. Compensation of input delay that depends on delayed input. Automatica, 85:362-373, 2017.

[16] J.-Y. Dieulot and J.-P. Richard. Tracking control of a nonlinear system with input-dependent delay. Proc. of the 40th Conference on Decision and Control, 4:4027-4031, 2001.

[17] D. Dochain. State and parameter estimation in chemical and biochemical processes: a tutorial. Journal of process control, 13(8):801-818, 2003.

[18] K. Gu and S. I. Niculescu. Survey on recent results in the stability and control of time-delay systems. Journal of Dynamic Systems, Measurement, and Control, 125:158, 2003.

[19] J. Harmand and D. Dochain. The optimal design of two interconnected (bio) chemical reactors revisited. Computers \& chemical engineering, 30(1):70-82, 2005.

[20] M. Jankovic. Recursive predictor design for linear systems with time delay. In American Control Conference, pages 4904-4909, 2008

[21] I. Karafyllis and M. Krstic. Numerical schemes for nonlinear predictor feedback. Mathematics of Control, Signals, and Systems, 26(4):519546, 2014.

[22] V. Léchappé, E. Moulay, and F. Plestan. Prediction-based control for lti systems with uncertain time-varying delays and partial state knowledge. International Journal of Control, 91(6):1403-1414, 2018.

[23] O. Lepreux, Y. Creff, and N. Petit. Model-based temperature control of a diesel oxidation catalyst. Journal of Process Control, 22(1):41-50, 2012

[24] T. Leroy, J. Chauvin, and N. Petit. Motion planning for experimental air path control of a variable-valve-timing Spark Ignition engine. Control Engineering Practice, 17(12):1432-1439, 2009.

[25] A. Manitius and A. Olbrot. Finite spectrum assignment problem for systems with delays. IEEE Transactions on Automatic Control, 24(4):541-552, 1979

[26] F. E Marble and D. W Cox Jr. Servo-stabilization of low-frequency oscillations in a liquid bipropellant rocket motor. Journal of the American Rocket Society, 23(2):63-74, 1953.

[27] J. Meyer, S. Midlam-Mohler, and Yurkovich S. In-cylinder oxygen concentration estimation for diesel engines via transport delay modeling. In American Control Conference, 2011.

[28] W. Michiels and S. I. Niculescu. Stability and Stabilization of TimeDelay Systems. Society for Industrial and Applied Mathematics, 2007.

[29] S. Mondié and W. Michiels. A safe implementation for finite spectrum assignment: robustness analysis. In Proc. of the 42nd IEEE Conference on Decision and Control, 2003.

[30] M. T. Nihtila. Finite pole assignment for systems with time-varying input delays. In Proc. of the 30th IEEE Conference on Decision and Control, pages 927-928, 1991.

[31] R. H. Perry, D. W. Green, and J. O. Maloney. Perry's Chemical Engineers' Handbook, volume 7. McGraw-Hill New York, 1984.

[32] J.-P. Richard. Time-delay systems: an overview of some recent advances and open problems. Automatica, 39(10):1667-1694, 2003.

[33] O. J. M. Smith. A controller to overcome dead time. ISA Journal, 6(2):28-33, 1959

[34] H. S. Tsien. Servo-stabilization of combustion in rocket motors Journal of the American Rocket Society, 22(5):256-262, 1952. 
[35] J. J Winkin, D. Dochain, and P. Ligarius. Dynamical analysis of distributed parameter tubular reactors. Automatica, 36(3):349-361, 2000.

[36] E. Witrant. Stabilisation des systèmes commandés par réseaux. $\mathrm{PhD}$ thesis, Laboratoire d'Automatique de Grenoble, 2005.

[37] E. Witrant, A. D'Innocenzo, G. Sandou, F. Santucci, M. D. Di Benedetto, A. J. Isaksson, K. H. Johansson, S.-I. Niculescu, S. Olaru, E. Serra, S. Teninna, and U. . Tiberi. Wireless ventilation control for large-scale systems: The mining industrial case. International Journal of Robust and Nonlinear Control, 20(2):226-251, 2010.

[38] K. Zenger and A. J. Niemi. Modelling and control of a class of time-varying continuous flow processes. Journal of Process Control, 19(9):1511-1518, 2009. 